\title{
Multi-Radio Resource Management for Ambient Networks
}

\author{
Fredrik Berggren ${ }^{1)}$, Aurelian Bria $^{* 1}$, Leonardo Badia $^{2)}$, Ingo Karla ${ }^{3)}$, Remco Litjens ${ }^{4)}$, Per Magnusson ${ }^{5}$, Francesco Meago ${ }^{6}$, \\ Haitao Tang ${ }^{7)}$, Riccardo Veronesi ${ }^{2)}$ \\ Royal Inst. of Technology $(\mathrm{KTH})^{1)}$, Consorzio Ferrara Ricercha ${ }^{2)}$, Alcatel ${ }^{3)}, \mathrm{TNO}_{\mathrm{ICT}}{ }^{4)}$, Ericsson $\mathrm{AB}^{5)}$, Siemens ${ }^{6}$, Nokia $^{7}$
}

\begin{abstract}
The Ambient Networks concept targets forthcoming dynamic communication environments, characterized by the presence of a multitude of different wireless devices, radio access technologies, network operators and business actors, which can form instant inter-network agreements with each other. MultiRadio Resource Management (MRRM) mechanisms, coordinating several radio accesses, fulfill a key role for providing wireless services with improved resource efficiency, coverage and service quality. This paper presents an MRRM concept for Ambient Networks, describes the principal MRRM functions and discusses design criteria.
\end{abstract}

\section{INTRODUCTION}

In the future, a mixture of available heterogeneous radio access technologies (RATs) can be foreseen. In principle, this multi-radio access scenario opens the potential to provide access to any network in an "Always Best Connected" fashion [1], possibly also utilizing several access techniques simultaneously, associated with competing network operators. The Ambient Networks (AN) project [2] aims at an innovative, industrially exploitable new network vision based on dynamic coordination and integration between networks to avoid adding to the growing patchwork of extensions to existing architectures. This will provide access to any $\mathrm{AN}$, in some cases even without subscription, including public as well as private networks, through instant establishment of internetwork agreements and network compositions. These internetwork agreements can be the result of network collaboration and negotiation or even include external access brokers. The user-centric AN vision extends many of the traditional networking principles of today and has impact on both the system design, which is the focus of this paper, as well as the business models.

Research on how to combine different RATs within a single operator domain is published in [3], [4] and [5]. More related work is conducted in a number of EU IST FP projects, e.g., BRAIN, MIND, DRiVE, WINE, ARROWS, MONASIDRE, and EVEREST. However, this research has considered only partial issues towards full network collaboration at the radio access level. In order for this dynamic concept to become a reality, a generic multi-radio access (MRA) architecture [6] has been outlined, which should not only be able to handle several RATs, but also provide for the aforementioned novel ambient networking principles. This paper proposes a consistent framework for access selection and resource allocation by joint

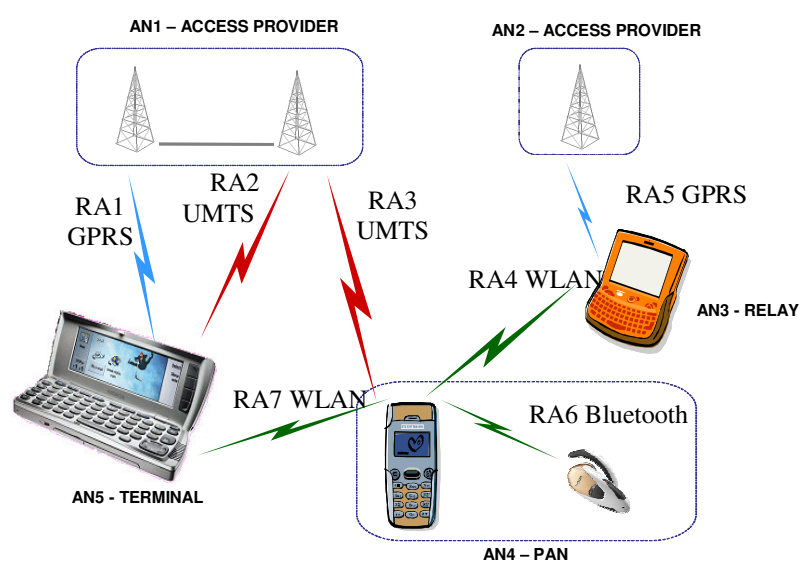

Figure 1. The Ambient Networks' multi-radio concept, in single and multihop case. AN5 maintains a data flow to AN1 dynamically shared over both RA1 and RA2, and a second flow towards AN4 by means of a direct communication. AN4 and AN2 communicate by relaying through AN3.

radio resource management across different RATs, and, networking support for more access providers and operators which enable new business relationships among involved actors, according to the AN paradigm.

The MRA architecture, [7] and [8], which is a key part of the AN concept, enables networks utilizing several access techniques to communicate, see Fig 1 . Since a key property of ANs is their ability to compose, they can form a new AN, with fully, or partially, shared network control. On the radio access (RA) level, the MRA architecture consists of the Multi-Radio Resource Management (MRRM) and the Generic Link Layer (GLL). The MRRM is responsible for joint management of radio resources between the different RAs with focus on system, session and flow level aspects. The GLL provides unified link layer processing, offering a generic interface towards higher layers and an adaptation to the underlying RATs [9].

The rest of the paper is organized as follows. In Section II, we introduce the logical concept of MRRM and describe the architecture. Section III gives a thorough overview of the MRRM functions. Section IV outlines the distribution and composition opportunities to be provided by the MRRM. The conclusions in Section $\mathrm{V}$ end the paper. 


\section{MRRM LOGICAL CONCEPT}

The gain from MRRM stems from mainly three principles; multi-access diversity, multi-access combining and dynamic multi-access management.

The multi-access diversity captures the possibility to have several RAs to choose from in the access selection. The multi access combining probes even further, by simultaneously combining transmission over several RAs. The multi-access management provides the common mechanism for an AN to dynamically add or remove a specific RA, which can be caused by change of composition, or simply by a device installation/removal and enabling/disabling to the AN.

\section{A. Benefits of MRRM}

The MRRM functionality aims at providing extended capacity and service coverage by 'merging' RA-specific capacity/coverage. Also, it should give enhanced overall resource efficiency by selecting the most efficient (combination of) RA(s) based on trade-offs between resource usage (spectrum, power etc.), costs, end- user preferences, QoS requirements, etc. Moreover, support of additional services that cannot be supported by individual RAs, by applying multi access combining, is crucial.

Moreover, to support the AN vision, the MRRM should handle resource management support for multi-hop networking, multi-operator cases, i.e., coordination between different administrative domains, and multicast/broadcast over multiple RAs.

\section{B. A Logical Concept Description of MRRM}

The MRRM concept is divided in two logical parts ( 1 and 2), to be built on already existing intrinsic RRM functions (3):

\section{RA coordination functions}

The scope of these generic functions spans over the available RAs and typically includes functions such as dynamic RA addition and removal, inter-MRRM communication, RA selection, inter-RA handover, congestion control, load sharing, adaptation of the allocated resources in a coordinated manner across several available RAs, etc.

\section{Network-complementing RRM functions}

These technology-specific functions are particularly designed for one or more RAT(s). However, these functions do not replace the existing RRM functions for the RAT(s) but rather complement them. These functions may:

- Provide missing, or complement inadequate, RRM functions to an underlying RAT, e.g., providing admission control, congestion control, intra-RAT handover to IEEE 802.11 based WLAN, which does not feature such functions.

- Be responsible for the RAT-specific interaction of the RA coordination functions and act as an adaptation function towards the network-intrinsic RRM functions. Hence, they appropriately translate format/terminology or commands to support effective interaction.
3. Network-intrinsic RRM functions

These are the basic RRM functions on which MRRM functions are built/mapped and they may belong to legacy as well as future systems.

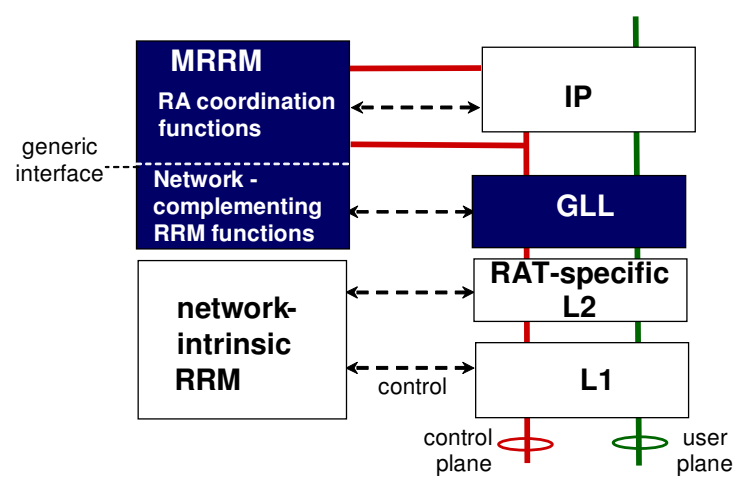

Figure 2. The Ambient Networks multi-radio architecture.

Some network-intrinsic RRM functions of existing legacy networks may already include RA coordination functions (e.g., load sharing and RA selection as developed in 3GPP), although not specifically developed within the AN context. Such functions can be utilized by the RA coordination functions. A clear split between the RA coordination functions and the network-complementing RRM functions is achieved by a standardized and generic interface.

\section{MRRM in the MRA Architecture}

A high-level model of the MRA architecture is depicted in Fig. 2, including the MRRM and the GLL and their position in the protocol stack. The GLL is a generic toolbox of link layer functions, which provides a unified interface to higher layers and facilitates efficient inter-working among multiple, possibly diverse, RAs. The proposed GLL, which is described further in [9], enables two novel concepts. The first of these, named Multi-Radio Transmission Diversity (MRTD), implies the sequential or parallel use of multiple RAs for the transmission of a traffic flow. The second, termed Multi-Radio Multi-Hop networking (MRMH), implies link layer support for multiple RAs along each wireless connection over a multi-hop communication route.

The GLL may be controlled by the MRRM, but the MRRM should be able to work independently of the GLL (for modularity reasons). For this, a standard interface needs to be specified between the MRRM and other functions in the MRA architecture, see [6] for more details on interfaces.

\section{MRRM FUNCTIONS}

MRRM is a control plane functionality which operates at system, session and flow level. At the system level, MRRM performs, e.g., spectrum, load and congestion control across two or more RAs. At the session level, MRRM coordinates decisions on different associated flows, where MRRM operations can be triggered either by system level operations or directly by session/flow level events, e.g., session arrivals or 
mobility. At the flow level, MRRM establishes and maintains RAs that are possibly constituted of parallel multi-hop routes.

The MRRM works through the establishment and maintenance of different RA sets:

- $\quad$ MRRM Detected Set (MRRM DS) is the set of all RAs that have been detected by the MRRM.

- $\quad$ MRRM Candidate Set (MRRM CS) is the set of RAs that are candidates to be assigned by the MRRM to a given data flow; it is always a flow-specific subset of the MRRM DS.

- $\quad$ MRRM Active Set (MRRM AS) is the set of RAs assigned by the MRRM to a given data flow at a given time, and is always a subset of the MRRM CS.

- GLL Active Set (GLL AS) is the set of RAs assigned to a given GLL entity by the MRRM to serve a given data flow at a given time; it is always a subset of the MRRM AS.

Information included in each set may cover RA identity, capabilities, related measurements, access costs etc. The set definitions are valid in single-hop as well as multi-hop cases, combining multiple RAs sequentially (multi-hop) and/or in parallel. The operational time scale for the GLL AS and MRRM AS updates depends on implementation and deployment aspects such as processing capabilities, transmission rate etc., as well as RAT specific limitations. The RAs in the GLL AS can be reconfigured autonomously without MRRM intervention. This would be useful for fast link adaptation techniques, e.g., MRTD.

\section{A. Session/flow level RA coordination functions}

For dynamic networking and business solutions, it is essential to advertise bandwidth and/or other network resources, including both public and private operators. Such RA advertisements should give sufficient adequate information for the MRRM and could serve as a basis for starting negotiations in a composition process. $R A$ Advertisement informs about the presence of a network or its capabilities to provide a given service, possibly in a business oriented fashion (with associated costs). To provide advertisements on behalf of other access providers or network nodes, proxy advertisements could be sent. RA advertisements are novel and crucial in achieving the vision of competitive and dynamic ANs.

The RA Discovery function may use the RA Advertisements to identify and monitor candidate RAs and routes for specific flows. Thereby it establishes and maintains the MRRM DS and MRRM CS. Along with other information, the discovered RAs are used for deciding which RAs and paths shall be included in the MRRM CS for the flow. Other information can be user and application preferences (QoS, security etc), dynamic composition agreements, network topology information etc. Both passive scanning, where nodes listen for RA advertisements sent over the air using beacons, pilots etc., on dedicated or broadcast channels, and dedicated scanning, where nodes receive information of new RAs to scan for on an existing RA, should be supported.

The RA Selection function is used to select the MRRM AS and the GLL AS for different flows, based on the MRRM CS of each flow. The first step of the RA Selection process is an RA evaluation wherein several parameters may be considered, including: signal quality and strength, end-user QoS , end-user costs, multi-operator network capacity, RA capabilities (also for multiple hops), user and provider preferences and policies, and operator revenues in single/multi-operator scenarios. The evaluation is then followed by an $R A$ Admission decision, ensuring that already established QoS agreements are protected. If the admission fails, RA Admission may try to admit other selected RAs, or the whole RA Selection may need to be repeated (omitting the denied RAs). During RA Selection, the MRRM may also perform bearer selection, i.e., the MRRM should inform the GLL regarding the bearer information, i.e., the way the assigned RAs should be used, depicting the type of channel (e.g. shared versus dedicated) that has been assigned, and perhaps a maximum allowable rate. Figure 3. shows an simulation example of capacity gains (in terms of satisfied users) of RA Selection for two composed operators deploying DS-CDMA cellular systems, providing a service with $15 \mathrm{~dB}$ processing gain and an $E_{b} / I_{0}$ requirement of $5 \mathrm{~dB}$. These gains come at the expense of maintaining large RA sets, which requires frequent signaling.

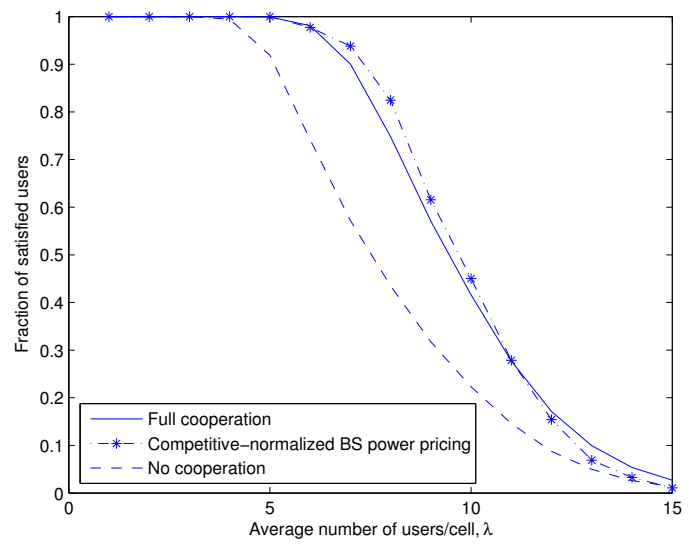

Figure 3. Achievable gain in fraction of satisfied users as a function of load

In the full cooperation case the users are assigned to the base station with the strongest path gain, regardless the user subscription. The reference case is when there is no cooperation between operators and users can connect only to the subscribed network. The third curve show the case when users choose freely which network to connect on the base of advertised information from each operator.

An application or a service may require multiple flows (voice, data, streaming, etc.), each with different QoS requirements. For flows belonging to the same application or service, the Session Management function makes coordinated session decisions for all flows. Therefore, no unnecessary resource reservations are performed by session selection due to that resources are only reserved when all flows within the 
session can be supported simultaneously. This can be done both across multiple RA as well as multiple hops. Moreover, if hard congestion actions are needed, this can be done in a more efficient manner, as it is better to remove all flows for targeted session for all hops. For admitting a session, the MRRM may have an option to consider splitting the session on several RAs, in such a way that the flows are allocated to the RAs which are most suited for the requirements of the flows. During the session's lifetime, different RA coordination functions ensure that all flows within the session continue to be assigned the appropriate RAs according to the QoS agreements and also considering the resource situation within the different RAs.

\section{B. System level RA coordination functions}

The system level RA coordination functions do not operate from a direct session/flow level perspective, but rather from the system perspective, monitoring and managing network load and resource efficiency.

Overall Resource Management is a functionality that keeps an overall control of the resources in order to perform congestion control, load sharing and spectrum control, in a proactive manner. The scope of the overall resource management functions covers multiple MRRM functions in multiple ANs. Overall resource management may employ different means in order to achieve its goal, resulting in different outcomes such as; flow and session dropping, QoS downgrading, (intra or inter-AN) handovers and resource reconfiguration within the system, followed by a number of RA Selection events for the affected flows.

As part of the overall resource management function, both soft- and hard Congestion Control are envisaged. The purpose of soft congestion control and Load Sharing is to detect upcoming congestion, where QoS agreements can no longer be honored, and take measures to prevent true congestion from occurring. In case soft congestion control fails to prevent true (hard) congestion, the hard congestion control function starts dropping flows/sessions in a controlled manner. In addition, soft congestion control can free up resources in support of a new session/flow request that the MRA system might wish to admit. It is noted that sometimes a congestion situation can be resolved within the own MRRM scope of a single AN. In such a case, the MRRM can take classical measures of existing radio systems to control its own radio resources by soft- and/or hard congestion control. In the AN world, however, where all nodes and terminals are AN aware and are able to negotiate in order to share their resources, a given AN node in or near congestion can communicate with neighboring nodes in order to jointly solve this congestion situation. Hence consideration of interAN measures, including inter-AN load sharing (directed handovers) or spectrum control (channel borrowing), will enhance the potential of preventing/resolving congestion. In this way, ANs can cooperate and help each other in congestion situations, or sell spare capacity, allowing a more efficient and flexible utilization of the available resources. This is a new AN capability, not yet present in current systems, which necessitates further research on charging and compensation schemes. Moreover, handover in an AN context could comprise change of RA, not only caused by mobility or other network related triggers such as congestion, but also due to that the user itself finds some more attractive access offer. This could cause "churning effects" [10], which affects the stability of the resource allocation.

Spectrum Control is the mechanism to coordinate and possibly (re)assign the frequency spectrum, within the same or different ANs, possibly across multiple RAs, e.g., to direct spectrum towards a RA that is most fit to handle the current traffic load and service mix. Such a dynamic mechanism may require allocation of bands with more liberalized frequency regulations which permit, e.g., secondary markets, lease of spectrum, change of use and non-exclusive frequency bands. Access to more spectrum can be done in different ways, e.g.:

- Having a shared band consisting of a common channel pool allowing dynamic channel allocation within one or between multiple ANs.

- Reassigning spectrum between multiple RATs.

For frequency bands that are shared between several ANs, e.g., described by the first bullet above, either the spectrum control within MRRM or an external access broker can take an active role coordinating the use of spectrum. Depending on the level of MRRM/GLL integration and composition format, control actions can potentially be taken on the scale of milliseconds, by means of fast channel allocations, to hours and days, planning away congestion in hot spots.

The second bullet describes the possibility to expand a frequency band by using spectrum dedicated to other RAs. They could be part of the own AN, or belong to other ANs and license holders. In the latter case, the release of carriers and channels from one to another AN, may be negotiated in the network composition phase.

\section{MRRM DISTRIBUTION ASPECTS}

\section{A. Distribution and Functional Split}

MRRM functionality may be distributed (logically and physically) within and in-between ANs in various ways. This will affect the type and amount of information that can be exchanged, the time scale at which information exchange is feasible, and thus the possible degree of coordination. Since the MRRM uses different sources of network information as input, e.g., radio-, security or business related, finding the best location of an MRRM entity is non-trivial.

Centralized MRRM control is likely to be beneficial in any network composed by a number of ANs belonging to a same administrative entity, e.g., a personal area network (PAN). Distributed solutions could be used in large networks for scalability reasons, or when a central coordination is simply not desirable due to the fact that the involved administrative entities pursue different strategies or do not trust each other. Multi-hop solutions may also fall in this category. Scenarios characterized by a large number of small networks without apriori (trust) relations are believed to put high requirements on the negotiation of MRRM roles.

When discussing how to distribute MRRM functions in a network it is important to consider not only desired traffic 
characteristics but also how radio resources, corresponding measurement statistics and control parameters are distributed over the network. From a control point of view, it is of course beneficial to place the MRRM functions where the measurement statistics and/or control originate. Further studies are needed to investigate protocol delays and outdated info.

\section{B. Network Composition and MRRM}

The role of a MRRM function should be defined during the negotiation process when ANs compose. In principle, an AN may consist of only one user terminal up to the case of a whole cellular network, which highlights the need for a general architecture. The MRRM roles can also be renegotiated dynamically at a later point in time, e.g., under the influence of changes in the external situation. One important aspect of the negotiation is to choose a strategy to follow (or a priority list of aspects to consider). Potentially, conflicting strategies may occur, e.g., optimizing power consumption, network capacity, sharing revenues among networks, etc. As an outcome, one MRRM entity may take a more dominant role (or master role), where MRRM coordinates resources by either, providing MRRM decisions or, providing MRRM co-ordination information. Alternatively, with a more passive role (or agent role), the MRRM may as a minimum, provide information to other MRRM functions that will facilitate the actual coordination. In addition, a passive MRRM may be able to request MRRM co-ordination information or decisions from a suitable MRRM function.

\section{Aspects of Multi-Operator MRRM}

One of the novel properties, coming as a consequence of the user-centric AN vision, is an increased level of competition for radio resources. The MRRM may experience two different situations; competitive actors or cooperative actors.

The first situation corresponds to a competitive market where operators do not reveal or exchange network information. Composition of networks may be hindered by competition or disagreements between actors. Efficient MRRM cannot be implemented without the help of a third party. Access brokers can be crucial, replacing the need for a direct composition between operators by acting as mediators and trusted parties. They will interact at high system level with the resources made available by each operator. Competitive MRRM will be highly related to game theory [10]. As an example, Figure 3. contains a plot of competitive MRRM, where the operators compete and use congestion pricing. The results show that there are gains similar to full cooperation case, but these come at the expense of more unstable behavior (not shown here).

Under the cooperative case the operators can establish business/trust relations and exchange MRRM data (user data, load status, etc.). Operators strive to agree on the roles that each of them is playing in the resource management. Note here that due to the high cooperation of all constituents of the composed networks, all the disadvantages mentioned in the previous section could be mitigated. This results in high capacity utilization of the overall composed network, better RA selection due to unbiased and complete input information and also conflict resolution when choosing a specific RA due to the possible different optimization criteria. Finally, the complete set of services from both operators is available in the coverage area where at least one of the operators can provide sufficient coverage.

\section{CONCLUSIONS}

In this paper, a functional description has been given for the MRRM in ANs, supporting MRA and transmission over different administrative entities and multi-hop links. Hence, the design needs to be flexible and applicable to PANs and body area networks (BANs), as well as up to large public operator networks. This work extends previous approaches on multiRAT systems by also considering cross-domain issues and negotiation of MRRM roles and features. In particular the architecture should support new types of access providers, e.g., access brokers and privately deployed networks. By negotiations, ANs should be able to compose and share resources more efficiently. Ongoing studies aim to verify the feasibility of the outlined MRRM concepts, as well as the potential gains in terms of capacity, coverage and service quality. In particular competitive MRRM contains many challenges, but also finding the best functional split of MRRM functionalities under different network configurations, using mobility triggers based on advertised network information, algorithm development and implementation issues and migration paths from existing technologies.

\section{ACKNOWLEDGMENT}

This paper describes work undertaken in the context of the Ambient Networks project, which is part of the EU's IST program. In total 41 organizations from Europe, Canada, Australia and Japan are involved in this Integrated Project, which will run from 2004-2005 in its first phase. The views and conclusions contained herein are those of the authors and should not be interpreted as necessarily representing the Ambient Networks Project.

\section{REFERENCES}

[1] E. Gustafsson and A. Jonsson, "Always best connected," IEEE Wireless Communications, vol. 10 , no. 1, pp. 49-55, 2003.

[2] WWI Ambient Networks, http://www.ambient-networks.org.

[3] A. Furuskär and J. Zander, "Multiservice allocation for multiaccess wireless systems," IEEE Trans. Wireless Commun., vol.4, no.1, 2005.

[4] A. Tölli, P. Hakalin, and H. Holma, "Performance of common radio resource management (CRRM)," in Proc. IEEE ICC, vol. 5, 2002.

[5] F. Malvasi et al., Traffic control algorithms for a multi access network scenario comprising GPRS and UMTS," in Proc. IEEE VTC Spring, vol. 1, pp. 145-149, 2003.

[6] N. Niebert et al., "Ambient Networks: A Framework for Future Wireless Internetworking," IEEE Vehicular Technology Conference - Spring, Stockholm, Sweden, May 2005

[7] J. Sachs et al., "Future wireless communication based on multi-radio access," in Proc. WWRF11, Oslo, Norway, 2004.

[8] J. Lundsjö et al., "A multi-radio access architecture for ambient networking," submitted to IST Mobile and Wireless Communications Summit, June 2005.

[9] K. Dimou et al., "Generic Link Layer: A solution for multi-radio transmission diversity in communication networks beyond 3G," to appear at IEEE VTC Fall 2005.

[10] S. K. Das and M. Chatterjee, "An econometric model for resource management in competitive wireless data networks," IEEE Network, vol. 18 , no. 6, pp. 20-26, 2004. 